\title{
Audit sur la qualité de la vaccination en milieu scolaire dans la région de Monastir (Tunisie)
}

\author{
Ines Bouanene, ${ }^{7}$ Faouzia Trimech, ${ }^{2}$ Arwa Ben Salah, ${ }^{7}$ Kamel Ben Salem Kamel ${ }^{7}$ et Sana El Mhamdi ${ }^{1}$
}

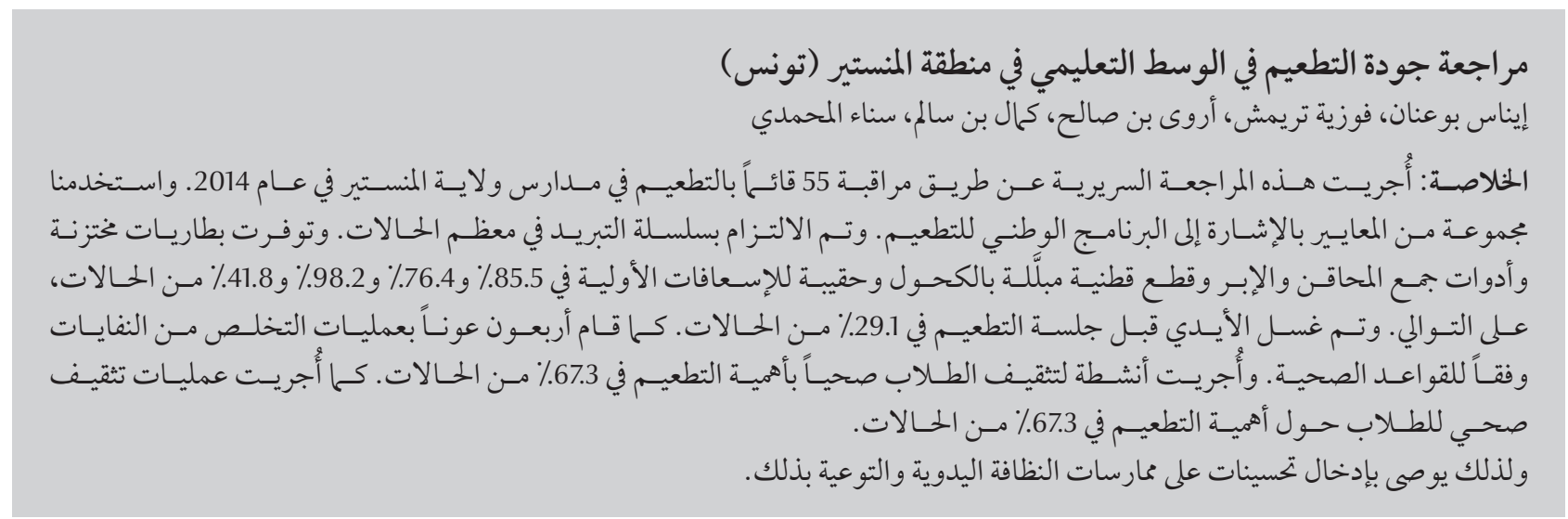

ABSTRACT We conducted a clinical audit by observing 55 vaccinators in schools in the governorate of Monastir in 2014. We used a set of criteria by referring to the national immunization programme. The cold chain was respected in most cases. Accumulators, the collector of syringes and needles, cotton balls soaked in alcohol and emergency box were available in $85.5 \%, 76.4 \%, 98.2 \%$ and $41.8 \%$ of cases respectively. Hand washing before immunization session was performed in $29.1 \%$ of cases. Waste disposal according to the hygiene rules was done by 40 agents. Health education of students regarding the interest of vaccination was done in $67.3 \%$ of cases. Therefore, improvements in hand hygiene practice and education are recommended.

RÉSUMÉ Nous avons mené un audit clinique par l'observation de 55 agents vaccinateurs dans les établissements scolaires du gouvernorat de Monastir en 2014. Nous avons utilisés un ensemble de critères en nous référant au programme national de vaccination. La chaîne du froid était respectée dans la majorité des cas. Les accumulateurs, le collecteur de seringues et aiguilles, les tampons de coton et d'alcool et la boîte d'urgence étaient disponibles dans $85,5 \%, 76,4 \%, 98,2 \%$ et 41,8 \% des cas respectivement. Le lavage des mains avant la séance de vaccination était effectué dans 29,1\% des cas. L'élimination des déchets selon les règles d'hygiène était réalisée par 40 agents. L'éducation sanitaire des élèves vis-à-vis de l'intérêt de la vaccination était faite dans 67,3\% des cas. Il est donc recommandé d'améliorer les pratiques d'hygiène des mains et l'éducation en la matière.

\footnotetext{
${ }^{7}$ Département de Médecine communautaire, Faculté de Médecine de Monastir, Université de Monastir (Tunisie) (Correspondance à adresser à I. Bouanene : bouaneneines@gmail.com). 2Direction régionale de Médecine scolaire et universitaire, Monastir (Tunisie).

Reçu : 16/07/16; accepté : 4/12/16
} 


\section{Introduction}

En Tunisie, la vaccination a été initialement introduite sous forme de campagnes de masse qui permettaient de faire baisser l'incidence des maladies cibles. Cependant, devant la persistance de bouffées épidémiques, un Programme national de Vaccination (PNV) a été mis en place en 1979. Il cible essentiellement les enfants d'âge préscolaire et scolarisés, avec des doses de rappel en milieu scolaire la première année de l'enseignement de base (vaccin antipoliomyélitique oral $[\mathrm{VPO}]$ et vaccin antirougeoleuxantirubéoleux $[\mathrm{RR}]$ ), la deuxième année del'enseignement debase (vaccin contre la diphtérie et le tétanos $[\mathrm{dT}]$ ), la sixième année de l'enseignement de base $(\mathrm{dT}+\mathrm{VPO}+\mathrm{RR})$, puis en troisième année d'enseignement secondaire $(\mathrm{dT}+\mathrm{VPO})(1)$.

Grâce au PNV, l'objectif d'une couverture vaccinale d'au moins $90 \%$ à l'échelle nationale a été réalisé pour les vaccins inclus dans le calendrier vaccinal (2). Dans la région sanitaire de Monastir, la vaccination en milieu scolaire a atteint cet objectif quantitatif avec des taux de couverture vaccinale élevés ( $\geq 97 \%$ ) (2). Cependant, on ne dispose pas de données qui explorent l'aspect qualitatif de la vaccination.

Ainsi, nous avons mené cette étude qui avait pour objectif de faire l'état des lieux de la qualité de l'acte vaccinal en milieu scolaire dans la région de Monastir.

\section{Méthodes}

\section{Type, lieu et population d'étude}

Il s'agit d'une étude descriptive transversale moyennant un audit d'observation externe, réalisée entre février et avril 2014 dans différents établissements scolaires (écoles et lycées) du gouvernorat de Monastir. Nous nous sommes attachés à évaluer la qualité de la vaccination faite par les agents vaccinateurs aux élèves en première, deuxième et sixième années de base, et en troisième année de secondaire. Nous avons récupéré les planning des visites pour la vaccination dans les établissements scolaires de toutes les circonscriptions de la région. Ayant à notre disposition, la liste exhaustive des agents au nombre de 55, 41 établissements ont été retenus pour l'étude.

\section{Collecte des données et variables de l'étude}

Trois auditeurs externes ont été recrutés pour réaliser ce travail, lesquels avaient été préalablementforméssurlesobjectifs et le déroulement détaillé de l'enquête afin de garantir un recueil standardisé des données. Ils ontété répartis sur les 11 circonscriptions sanitaires de la région. Chacun d'eux était chargé d'observer l'agent vaccinateur lors de son passage à l'école ou au lycée. Ces auditeurs ont programmé leurs sorties selon les données des organigrammes de vaccination. Chaque agent était observé lors d'une seule séance vaccinale.

En se référant au PNV (1), nous avons construit notre propre grille d'audit qui explore essentiellement le respect de la chaîne du froid, la disponibilité du matériel nécessaire à la séance vaccinale, le respect des règles d'hygiène et l'éducation sanitaire.

\section{Résultats}

L'audit a été réalisé dans 36 écoles primaires et cinq lycées secondaires (Tableau 1). Parmi les 55 agents audités, 51 déclaraient avoir informé le directeur de chaque établissement du programme vaccinal préétabli, suivant lequel 50 sujets avaient amené les doses de vaccin nécessaires pour chaque séance vaccinale.

Concernant le respect de la chaîne du froid (Tableau 2), la quasi-totalité des agents vaccinateurs recevaient les vaccins dans une glacière $(\mathrm{n}=54)$. À la réception des vaccins $\mathrm{VPO}$, seulement trois agents vérifiaient que la date de décongélation était inférieure à six mois. La vérification des dates d'ouverture des flacons de vaccin était effectuée pour le VPO et le dT par 35 agents vaccinateurs, et pour le RR par 32.

La plupart des agents vaccinateurs disposaient du matériel nécessaire à la séance vaccinale tel que le plateau

\begin{tabular}{|c|c|c|c|c|}
\hline Établissements scolaires & $\begin{array}{c}\text { Effectif des } \\
\text { établissements }\end{array}$ & $\begin{array}{l}\text { Effectif des agents } \\
\text { vaccinateurs }\end{array}$ & Type de vaccin & Niveau scolaire \\
\hline \multirow[t]{3}{*}{ École primaire } & \multirow[t]{3}{*}{36} & 19 & $V P O+R R$ & $1^{\text {re }}$ année de base \\
\hline & & 16 & dT & $2^{\mathrm{e}}$ année de base \\
\hline & & 13 & $V P O+R R+d T$ & $6^{\mathrm{e}}$ année de base \\
\hline Lycée secondaire & 5 & 7 & $d T+V P O$ & $3^{\mathrm{e}}$ année secondaire \\
\hline Total & 41 & 55 & - & - \\
\hline
\end{tabular}

$V P O=$ vaccin oral contre la poliomyélite $; d T=$ vaccin contre la diphtérie et le tétanos $; R R=$ vaccin antirougeoleux-antirubéoleux 


\begin{tabular}{|c|c|c|}
\hline Critères & $\begin{array}{l}\text { Effectif } \\
(n=55)\end{array}$ & $\%$ \\
\hline Réception des vaccins dans une glacière & 54 & 98,2 \\
\hline Vérification que la date de décongélation des flacons de VPO $<6$ mois & 3 & 5,5 \\
\hline Vérification que si un flacon est entamé, sa date d'ouverture $<15$ jours pour le VPO et dT & 35 & 63,6 \\
\hline Vérification que si un flacon est entamé, sa date d'ouverture $<6$ heures pour le RR & 32 & 58,2 \\
\hline Disponibilité d'un plateau contenant des accumulateurs spéciaux de froid sur la table & 47 & 85,5 \\
\hline Disponibilité d'un collecteur pour les seringues et aiguilles à usage unique utilisées & 42 & 76,4 \\
\hline Disponibilité des tampons de coton et de l'alcool sur la table & 54 & 98,2 \\
\hline Disponibilité d'une boîte d'urgence & 23 & 41,8 \\
\hline Port d'un tablier & 44 & 80 \\
\hline Bien se laver les mains avant la séance vaccinale & 16 & 29,1 \\
\hline Bien se laver les mains après l'acte vaccinal & 3 & 5,5 \\
\hline Assurer une bonne désinfection de la peau au point d'injection & 52 & 94,5 \\
\hline Siège de vaccination respecté & 51 & 92,7 \\
\hline Éviter de recapuchonner les aiguilles & 46 & 83,6 \\
\hline Placer les seringues et les aiguilles utilisées dans un collecteur & 40 & 72,7 \\
\hline Ramener ces déchets au CSB pour s'en débarrasser & 41 & 74,5 \\
\hline Passer quelques messages éducatifs sur l'intérêt de la vaccination & 37 & 67,3 \\
\hline Expliquer l'importance de bien conserver la carte de vaccination & 23 & 41,8 \\
\hline Prévenir et dépister les MAPI & 44 & 80 \\
\hline
\end{tabular}

$C S B=$ Centre de Santé de Base $; M A P I=$ manifestations postvaccinales indésirables .

contenant des accumulateurs spéciaux de froid, des tampons de coton et de l'alcool, les collecteurs pour les seringues et aiguilles à usage unique utilisées. En revanche, la boitte d'urgence était disponible pour 23 agents.

Concernant le respect des règles d'hygiène, le port d'un tablier était respecté dans $80 \%$ des cas. Le lavage des mains avec de l'eau savonneuse ou une solution hydroalcoolique était beaucoup moins observé chez les agents vaccinateurs, avec des proportions respectives de $29,1 \%$ avant la vaccination et 5,5\% après la vaccination. Au cours des séances vaccinales, 52 agents vaccinateurs assuraient une bonne désinfection de la peau au point d'injection et 51 respectaient le siège de la vaccination. Sur l'ensemble des 55 agents vaccinateurs, 46 évitaient de recapuchonner les aiguilles. Quarante sujets plaçaient les seringues et les aiguilles utilisées dans un collecteur lorsque celui-ci était disponible. Dans le cas contraire, des récipients en plastique contenant de l'eau de javel étaient utilisés par 15 agents. Par la suite, 41 infirmiers ramenaient les déchets au Centre de Santé de Base (CSB) pour s'en débarrasser.

À propos de l'éducation sanitaire en matière de vaccination, 37 agents passaient quelques messages éducatifs aux élèves sur l'intérêt de la vaccination. En revanche, l'éducation au sujet de la carte vaccinale, notamment l'importance de bien la conserver pour ne pas la perdre, n'était faite que par 23 agents, soit $41,8 \%$ des cas. Par ailleurs, la prévention et le dépistage d'éventuelles manifestations postvaccinales indésirables (MAPI) aiguës étaient effectués par $80 \%$ des agents.

Pour les 19 agents vaccinateurs audités avec les élèves de première année de base, 14 donnaient la carte de vaccination à ces élèves. Parmi les 36 séances vaccinales auditées dans les autres niveaux (deuxième et sixième années de base, et troisième année secondaire), la carte vaccinale n'était pas ramenée par les élèves lors de 32 séances. Dans ce cas, 14 agents vaccinateurs seulement remettaient une autre carte mise à jour à ces élèves.

\section{Discussion}

Le présent travail concernait l'évaluation des pratiques des professionnels de santé en matière de vaccination en milieu scolaire moyennant un audit d'observation directe. À notre connaissance, il n'y a pas d'études tunisiennes antérieures intéressant ce sujet. Pour cette raison, le choix de la région de Monastir, bien qu'il puisse être une source de biais de sélection, a été adopté par convenance pour tester la faisabilité de cette étude pilote. Afin de limiter les éventuels biais d'information, nous avons organisé des séances de formation des trois enquêteurs pour leur expliquer 
succinctement les différents items de la grille et la manière d'évaluer l'acte de façon uniforme, en se basant sur les recommandations du PNV et celles internationales, notamment en matière d'hygiène.

Dans notre étude, la quasi-totalité des agents vaccinateurs a amené les vaccins dans des glacières pour assurer les séances vaccinales. Cependant, la date de décongélation des flacons de VPO n'a été vérifiée que par trois individus. À noter que ce vaccin est le plus sensible à la chaleur : son stockage et son transport doivent se faire selon de bonnes pratiques de respect de la chaîne du froid $(3,4)$, chose qui n'a pas été observée dans notre travail. La présence d'accumulateurs spéciaux de froid munis de trous ou de briquettes congelées a été observée dans 85,5\% des cas. En fait, il est recommandé d'utiliser ces dispositifs en nombre suffisant et de les mettre dans un endroit exposé au vent afin d'éviter le problème de fonte (5). Nous avons noté aussi que près de $25 \%$ des agents vaccinateurs ne disposaient pas de collecteurs pour les seringues et aiguilles à usage unique, ce qui constitue un véritable problème vu le sérieux risque encouru en cas de piqûre accidentelle. Même si, en milieu scolaire, le risque infectieux est minime, il est capital de disposer de ce matériel. Au cours des séances vaccinales, près de $2 / 5$ des agents vaccinateurs seulement disposaient de la boîte d'urgence. La présence de cette dernière constitue une mesure de précaution pour faire face aux incidents qui pourraient survenir dans un endroit non médicalisé (tel que les établissements scolaires) à tout moment de la journée de vaccination, commel'anaphylaxie, la crise d'angoisse, la réaction vasovagale (6).

Le lavage des mains avant et après l'acte vaccinal était insuffisamment pratiqué par les agents audités. L'hygiène des mains constitue le premier défi mondial de l'Organisation mondiale de la Santé (OMS) pour la sécurité des patients (7). L'une des interventions préconisées dans ce cadre est la promotion d'une hygiène des mains optimale à observer lors d'une injection ou d'une vaccination (8). Cette défaillance observée dans notre travail pourrait être liée à la charge de travail, vu le nombre important d'élèves à vacciner par séance, ainsi qu'à la nondisponibilité d'une source d'eau et de savon dans les locaux conçus pour la vaccination dans les établissements scolaires. Ainsi, la mise à la disposition des agents vaccinateurs de solutions hydro-alcooliques pour le lavage des mains parait une bonne alternative en milieu scolaire. En effet, l'utilisation facile de ces produits permet, outrel'effet antiseptique, d'améliorer l’adhésion et l'observance des professionnels de santé au lavage des mains (9).

À la fin de l'acte vaccinal, le recapuchonnage des aiguilles a été observé dans 16,4\% des cas. Cette pratique, non conforme aux normes de sécurité, expose les agents vaccinateurs aux accidents d'exposition au sang. L'OMS exhorte les pays à passer, d'ici 2020, à l'usage exclusif des nouvelles seringues autobloquantes dites aussi « intelligentes » (10). Ces seringues, sur certains modèles, sont munies d'un clip métallique qui bloque le piston une fois qu'il est enfoncé. Sur d'autres, l'aiguille se rétracte dans le corps de la seringue à la fin de l'injection.

Dans notre étude, seulement 23 agents vaccinateurs ont expliqué aux élèves l'importance de bien conserver la carte de vaccination pour ne pas la perdre. En Suisse, la perte de la carte vaccinale, estimée à plus de $20 \%$ en 2015 (11), constituait l'élément justifiant l'élaboration d'un carnet de vaccination électronique qui a permis d'éviter la perte ou l'oubli du carnet de vaccination en papier.

Dans notre étude, les agents vaccinateurs ont essayé de passer quelques messages éducatifs sur l'intérêt de la vaccination de façon générale dans près de deux tiers des cas. Par ailleurs, les séances éducatives doivent être planifiées et programmées d'avance; elles peuvent se dérouler en trois temps : avant la vaccination sous forme de discussion de groupe, au moment de la vaccination sous forme d'entretien individuel, à la sortie sous forme de conseils (12).

\section{Conclusion}

Cette étude nous a permis de faire un constat global sur les pratiques des professionnels de santé en matière de vaccination en milieu scolaire. Elle a mis en évidence certains écarts observés qui nécessitent des interventions ciblées en vue d'une amélioration. Une attention particulière doit être apportée au respect de la chaîne du froid, vu l'importance qu' elle présente pour assurer la sécurité et l'efficacité des vaccins. De plus, la mise à la disposition des agents vaccinateurs du matériel nécessaire au bon déroulement d'une séance vaccinale est cruciale. Aussi, la sensibilisation de l'ensemble de ces agents au respect des règles d'hygiène et à la bonne pratique du lavage des mains est primordiale, surtout en encourageant l'utilisation des solutions hydro-alcooliques. Par ailleurs, la formation continue des professionnels de la santé en matière de vaccination est nécessaire pour renforcer leur adhésion aux recommandations de bonnes pratiques. Cette formation doit inclure également les techniques de communication vu le rôle important de ces agents dans l'éducation et la promotion de la santé.

Financement : aucun.

Conflit d'intérêt : aucun. 


\section{Références}

1. Programme National de Vaccination. Manuel à l'Usage des Professionnels de la Santé. Tunis (Tunisie): Ministère de la Santé publique, Direction des Soins de Santé de Base; 2002.

2. Programme National de Vaccination. Rapport annuel. Tunis (Tunisie): Ministère de la Santé publique, Direction des Soins de Santé de Base. 2009; 159-206.

3. Vaccination pratique: guide à l'usage des personnels de santé - mise à jour 2015. Genève: Organisation mondiale de la Santé; 2016. (http://apps.who.int/iris/ bitstream/10665/206455/1/9789242549096_fre.pdf?ua=1, consulté le 4 février 2016).

4. Mode d'emploi des pastilles de contrôle du vaccin - Facilité de gestion du vaccin pour les vaccinations supplémentaires de la polio. Genève: Organisation mondiale de la Santé, Département Vaccins et produits biologiques; 2000. (http://apps.who.int/iris/bitstream/10665/66586/1/WHO_ VB_00.14_fre.pdf, consulté le 4 février 2017).

5. Office fédéral de la santé publique, Commission fédérale pour les vaccinations. Plan de vaccination suisse 2016. Directives et recommandations. Berne: Office fédéral de la santé publique, 2016.

6. Morel V, Sarasin FP, Hauser C. Anaphylaxie : prise en charge initiale. Rev Med Suisse. 2005;29:30554.

7. Patient safety. WHO Guidelines on Hand Hygiene in Health Care. First Global Patient Safety Challenge Clean Care is Safer
Care. Geneva: World Health Organization; 2009 (http://apps. who.int/iris/bitstream/10665/44102/1/9789241597906_eng. pdf, consulté le 4 février 2017).

8. Organisation mondiale de la Santé. Un soin propre est un soin plus sûr - Les cinq volets du premier Défi mondial pour la sécurité des patients. (http://www.who.int/gpsc/elements/ $\mathrm{fr} /$, consulté le 4 février 2017).

9. Boyce JM. Update on hand hygiene. Am J Infect Control. 2013;41 Suppl 5:S94-6. 10.1016/j.ajic.2012.11.008

10. WHO guideline on the use of safety-engineered syringes for intramuscular, intradermal and subcutaneous injections in health-care settings. Geneva: World Health Organization; 2015 (http://apps.who.int/iris/bitstre am/10665/250144/1/9789241549820-eng.pdf, consulté le 4 février 2017).

11. Siegrist CA. Un carnet de vaccination électronique. Centre de Vaccinologie, Hôpitaux Universitaires de Genève (HUG), 2015 (http://www.hug-ge.ch/sites/interhug/files/ structures/medecine_de_premier_recours/formation mpr_geneve_septembre_2015_2e_version.pdf, consulté le 4 février 2017).

12. Broome ME. Preparation of children for painful procedures. Pediatr Nurs. 1990 Nov-Dec;16(6):537-41. 